\title{
Merging lidar with coarser DEMs for hydrodynamic modelling over large areas
}

\author{
$\underline{\text { John Gallant }^{\mathrm{a}}}$ \\ ${ }^{a}$ CSIRO Land and Water \\ Email:John.Gallant@csiro.au
}

\begin{abstract}
Hydrodynamic modelling of flood flows requires accurate topographic data, which in most cases means lidar or photogrammetric data with proper separation of ground and non-ground features like vegetation. The entire modelling domain must be represented but the area of focus is often a small part of the entire domain, so it is often cost-effective to use expensive and detailed elevation data in the focus area and cheaper, less detailed data elsewhere. This leads to the need for joining the two digital elevation models (DEMs) seamlessly so that there are no abrupt changes in height or slope at the transition.

In the Fitzroy River of Western Australia, an area of $37000 \mathrm{~km}^{2}$ was targeted for hydrodynamic modelling. Lidar data was collected in the floodplain area covering about $5800 \mathrm{~km}^{2}$ and the remaining area was covered by SRTM-derived DEM-H at 1 arcsecond resolution. A method for adjusting the DEM-H to match the lidar data was developed to remove abrupt steps at the boundary to ensure the combined data was suitable for hydrodynamic modelling.

The two main steps in the process were (1) removal of systematic vertical errors and (2) adjusting the less reliable DEM-H to match the lidar at the boundary. The method successfully removed local steps and produced a result suitable for hydrodynamic modelling. There is scope for extending the adjustment process to account for broader scale differences.
\end{abstract}

Keywords: Digital elevation models, lidar, blending 


\section{INTRODUCTION}

Hydrodynamic modelling of flood flows requires accurate topographic data, which in most cases means lidar or photogrammetric data with proper separation of ground and non-ground features like vegetation. The area being modelled often extends far beyond the area of real interest to avoid artificial boundaries that distort the modelling of flood flows, but lower accuracy topographic data can be used beyond the focus area without significantly influencing the results in the focus area. This allows use of low-cost or free digital elevation models (DEMs) to fill in the gaps where the investment in accurate DEMs is not cost-effective.

The less accurate DEM data will not join seamlessly to the higher resolution data. If the DEMs are simply merged without regard to height differences at the edges, there will be steps in elevation that substantially distort the modelled water flow and inundation extent in the area of interest. To prevent those problems, heights of one or both DEMs need to be adjusted near the boundary to produce a seamless join without steps and with as little slope distortion as possible.

The height differences occur at several different scales and due to various causes:

1. Less accurate and detail in the coarser DEM, leading to fine-scale differences related to topographic details, such as reduced magnitude of highs and lows for smaller features and the complete absence of finer details such as smaller channels

2. Broader scale variations due to a variety of errors in one or both data sources

3. Differences in horizontal or vertical datum leading to systematic shifts

This paper describes a method for producing a seamless join of high resolution lidar with lower resolution SRTM-based elevation data. The method deals with issues 1 (differences in detail) and 3 (datum shifts) and can be extended to include broader scale variations (issue 2). The method is suitable for use in applications other than hydrodynamic modelling, wherever a seamless join between different elevation models is needed to avoid distortion of the shape at the boundary.

\section{MATERIALS AND METHODS}

The area discussed is the floodplain of the Fitzroy River in Western Australia from Fitzroy Crossing to Willare, and its surrounds. This was one of several areas targeted for hydrodynamic modelling of flood flows as part of the Northern Australia Water Resource Assessment (NAWRA) project (Karim et al., 2018). The area of the modelling domain was about $35000 \mathrm{~km}^{2}$, a large area for hydrodynamic modelling.

Lidar was acquired at about 1 point per square metre and gridded at $5 \mathrm{~m}$ resolution, covering about $5800 \mathrm{~km}^{2}$ and focused on the floodplain. For the remainder of the area the best available data was the national hydrologically enforced DEM-H derived from SRTM (Gallant et al., 2011) at 1 arc-second resolution. The lidar data used the GDA94 MGA Zone 51 projection and AHD vertical datum, while DEM-H used WGS84 latitude-longitude coordinates and the EGM96 vertical datum.

\subsection{Resolution and datum adjustment}

As a first step the two DEMs were brought to a common projection and resolution. The lidar $5 \mathrm{~m}$ data was aggregated to $30 \mathrm{~m}$ by averaging groups of $6 \times 6$ cells. The DEM-H data was projected to MGA51 at the same $30 \mathrm{~m}$ resolution and with the same cell alignment. These steps were completed using ArcGIS.

There was no evidence of any horizontal misalignment. The GDA94 and WGS84 datums are very similar, with horizontal offsets of a few metres, and any differences are unlikely to be evident in $30 \mathrm{~m}$ resolution data (Intergovernmental Committee on Surveying and Mapping, 2014).

In northern Australia the SRTM elevations based on the EGM96 global geoid are higher than AHD mostly due to differences in the vertical datum; systematic errors in the SRTM heights also contribute to the differences. In the Fitzroy River area, the difference was found to be a fairly uniform $2.5 \mathrm{~m}$, so that amount was subtracted from the DEM-H elevations to align them (on average) with the lidar elevations. Removing the systematic error still leaves significant discrepancies at the boundary of the lidar data (Figure 1 and Figure 3a).

\subsection{Smoothing the transition}

Removing the abrupt change of elevation at the transition from lidar to DEM-H requires altering one or both DEMs around the transition. This could be achieved with a blending tool such as raster mosaic in ArcGIS, 
but this method alters both rasters in the overlap area; given the lidar data is more precise it should be left untouched, and all changes were applied to DEM-H. A method to achieve that was developed using Python with the NumPy and SciPy packages. The method uses a similar approach to the MBlend method (Leitāo et al., 2016) but is simpler and fully automated, requiring no user-defined set of points for generating the difference surface.

The first step in blending was to calculate the difference between the two DEMs where they overlap (Figure 2a). The difference values were then extended beyond the overlap area (into the area where DEM-H is the only source) by calculating the mean difference in a 3-cell square neighbourhood (using scipy.ndimage.uniform_filter), replacing nodata values beyond the overlap area with mean values; this extends the difference values by one cell beyond the overlap. This process was repeated once more to extend the values two cells $(60 \mathrm{~m})$ beyond the overlap.

Two further extrapolation steps were applied using Gaussian smoothing (scipy.ndimage.gaussian_filter) with standard deviations of 1.4 and 2 cells, extending the difference values about $400 \mathrm{~m}$ beyond the overlap (Figure 2b).

Using the uniform filter for the first two steps ensures the fine detail of differences is preserved immediately adjacent to the boundary. Earlier attempts using the Gaussian filter for all extrapolation steps produced too much smoothing of the difference near the edge, leaving obvious steps in the result.

The edge of the extended difference still had an abrupt transition from non-zero to zero values, so weighting values were calculated that smoothly changed from 1.0 at the original boundary to 0.0 at the edge of the extended difference. Multiplying the weighting with the extended difference produced the desired smooth transition to zero differences at the outer edge (Figure 2c).

The difference surface was subtracted from DEM-H to create an adjusted coarser resolution DEM that exactly matched the lidar elevations at the edge of the lidar extent. The adjusted DEM-H was refined to $5 \mathrm{~m}$ resolution to match the lidar and then merged with the lidar to create a single $5 \mathrm{~m}$ resolution DEM that was further processed for the hydrodynamic modelling (Karim et al., 2018). A combined $30 \mathrm{~m}$ resolution DEM was also produced to facilitate assessment of the surface and to produce the results shown below.

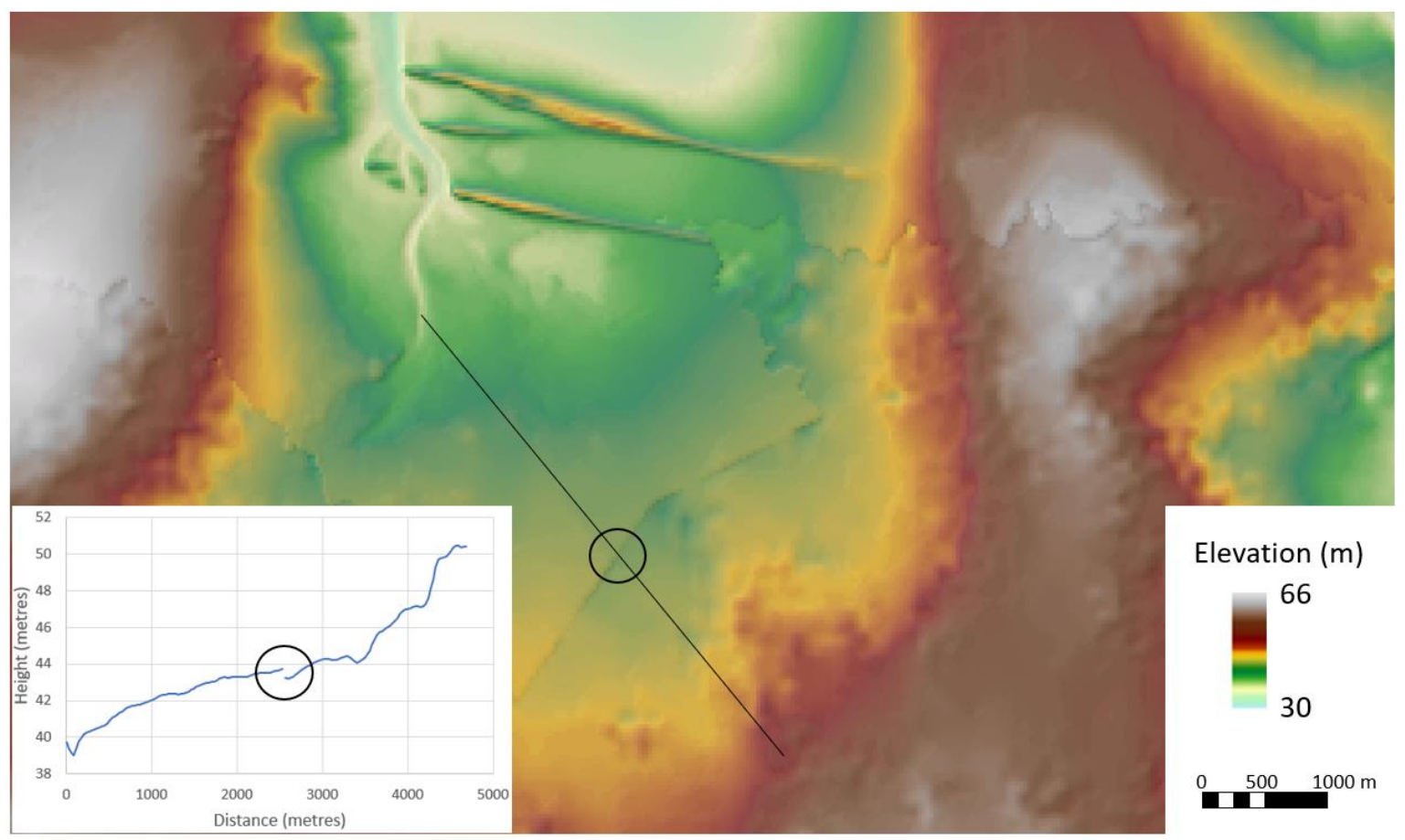

Figure 1. Lidar and DEM-H (both at $30 \mathrm{~m}$ resolution) after correction for vertical offset but before any treatment of edge offsets. The lidar data covers the upper part of the image and the DEM-H data is in the lower part. The black line shows the location of the cross-section in the lower left and the circles identify the location of the height discontinuity. 


\section{RESULTS}

Figure 3 shows the join between the two DEMs without and with the blending process, in the same area as the difference images in Figure 2. The obvious discrepancies at the boundary have been eliminated and, while the difference in detail is still very apparent, the exact location of the boundary is no longer obvious.

Figure 4 shows the area of Figure 1 with the blending process. The abrupt step at the boundary between the two DEMs has been eliminated producing a seamless transition, although there is still evidence of a broader scale offset where the lower resolution DEM-H has slightly lower elevations. The extent of this elevation offset is larger than the area covered by the extrapolated difference, so the blending process is not able to correct this offset.

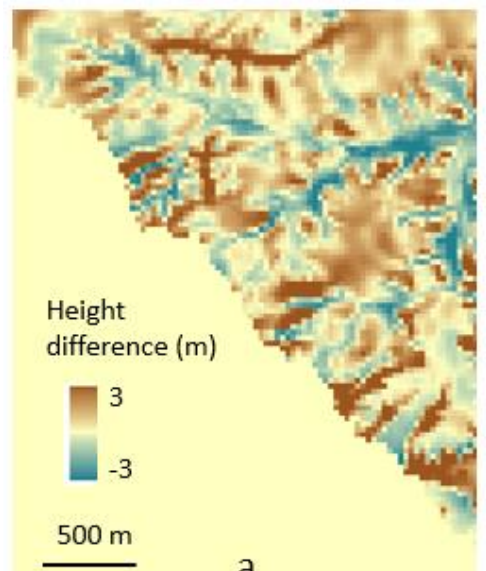

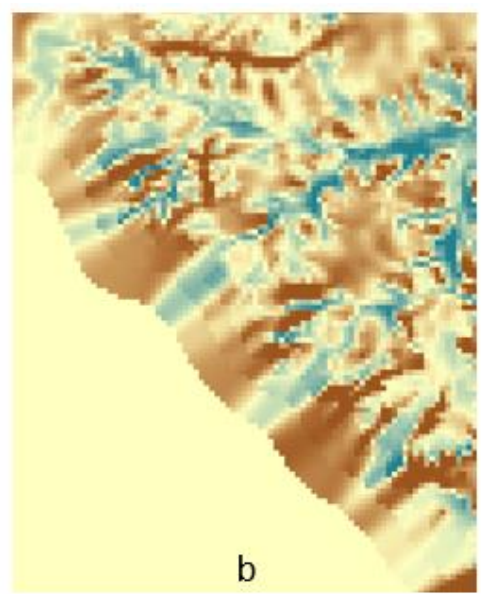

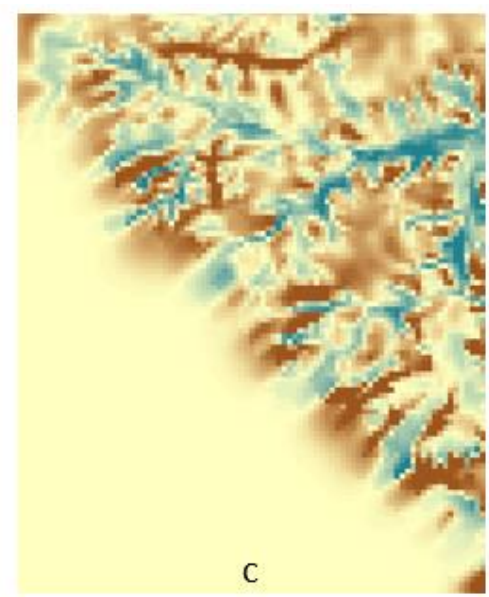

Figure 2. (a) The difference surface created by subtracting the $30 \mathrm{~m}$ resolution DEM-H from the 30 $\mathrm{m}$ resolution lidar. (b) The extended difference surface created by averaging over four progressively larger neighbourhoods. (c) The extended difference surface after multiplying by the smooth weighting function to reduce differences smoothly to zero at the outer edge.
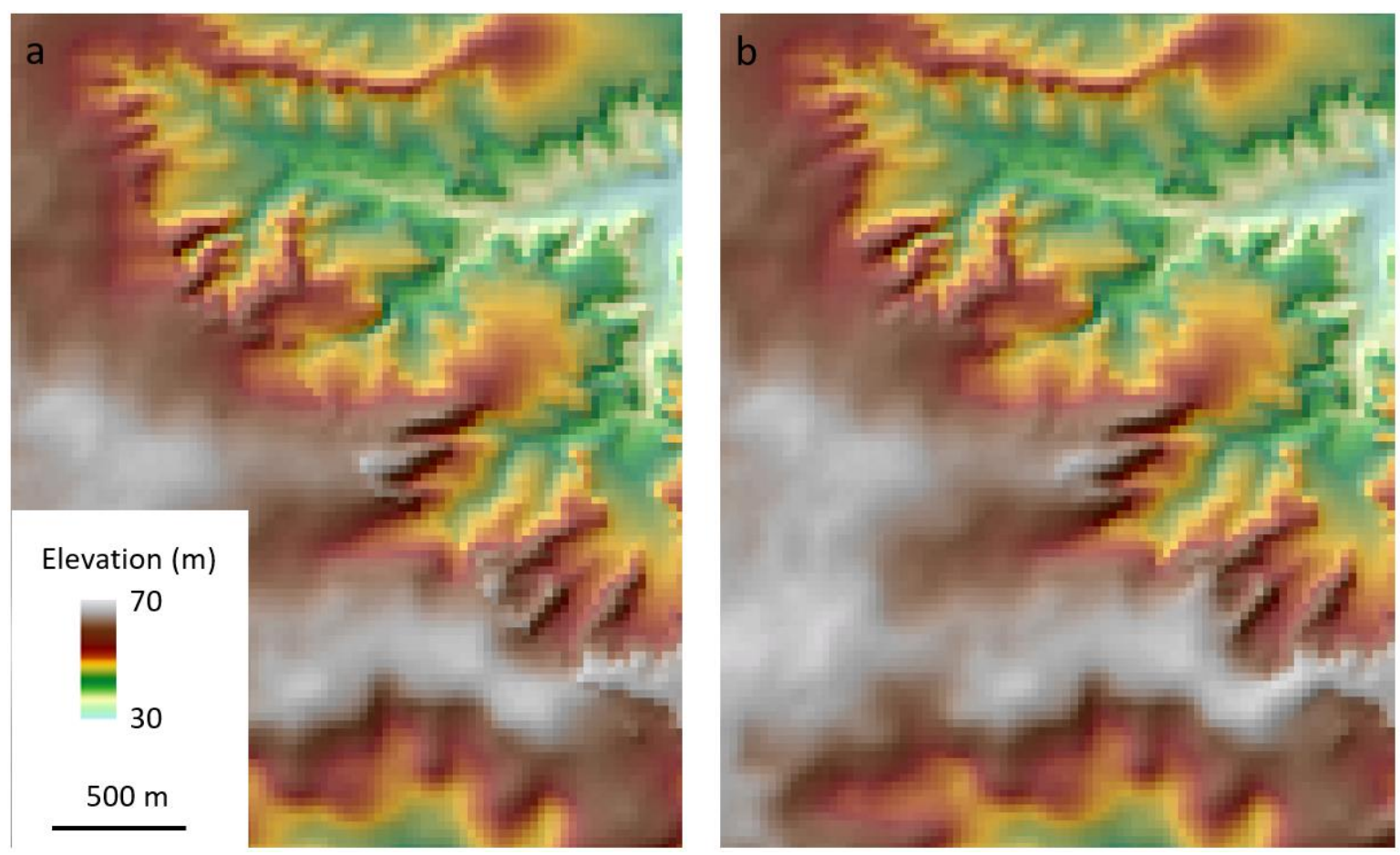

Figure 3. (a) Lidar and DEM-H at $30 \mathrm{~m}$ resolution showing mismatches due to lack of detail and inherent smoothing in DEM-H in the same area as Figure 2. The lidar elevations are higher on the ridges and lower in the valleys. (b) After blending, the transition from the lidar to DEM-H is smooth and has no abrupt steps. 


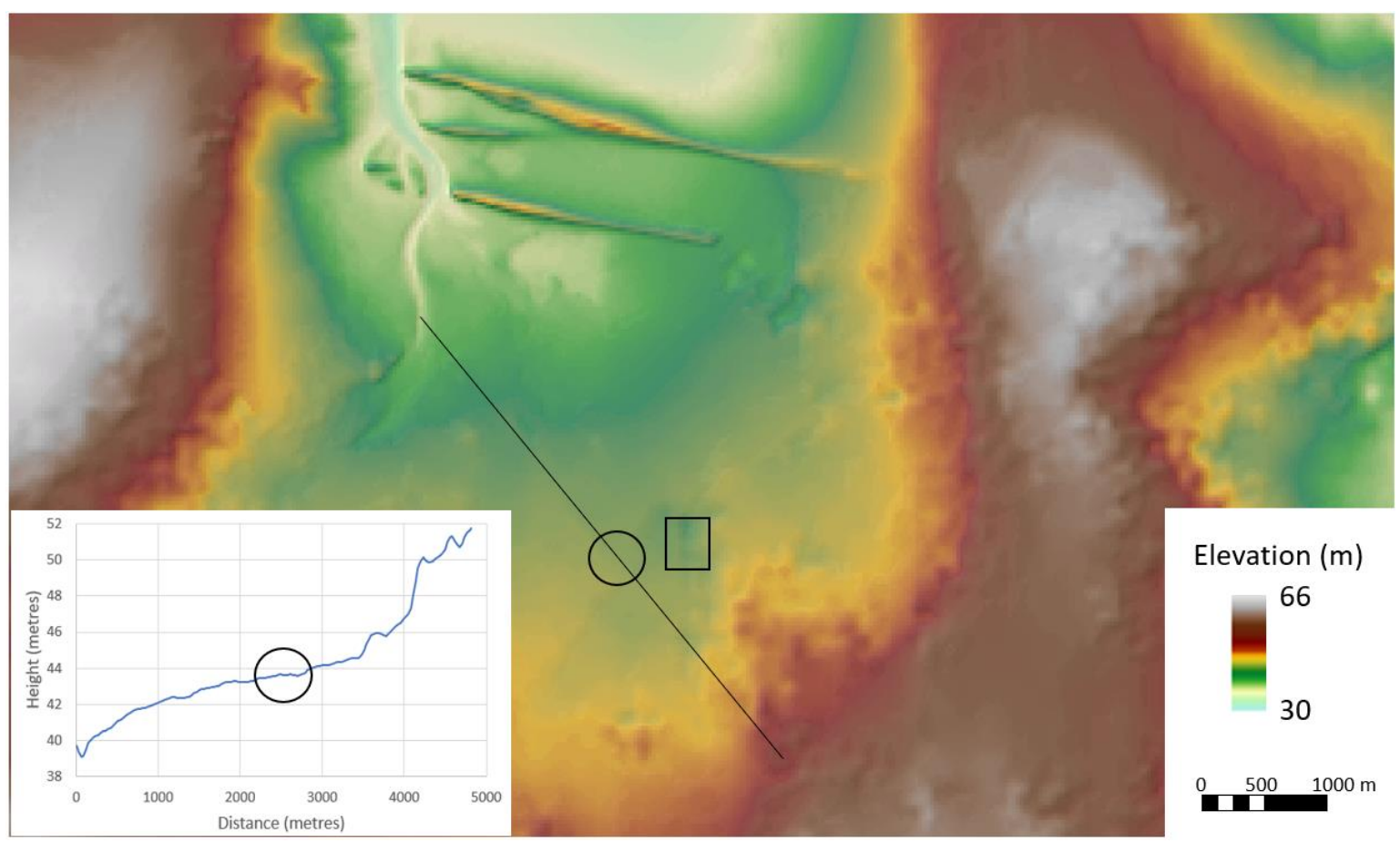

Figure 4. The blended lidar and DEM-H data of Figure 1 after treatment of edge offsets. The abrupt step visible in Figure 2 within the circles has been eliminated. The square shows an area where the elevation offset in the coarser DEM-H has remained untreated because it is outside the area covered by the extrapolated difference.

\section{DISCUSSION AND CONCLUSIONS}

The blending process has removed most of the differences between the lidar DEM and the SRTM-based DEM and produced a product that was considered to be suitable for hydrodynamic modelling. No attempt was made to assess the impact of the improved DEM on the hydrodynamic modelling due to the time constraints of the project and the effort required to duplicate the modelling.

In the Fitzroy River case, the height differences between the lidar and DEM-H were found to be consistent across the whole area. Where there are substantial spatial variations in the height differences that cannot be ignored, further steps can be taken to improve the results. As part of the same project, a blended DEM for the Mitchell catchment was also required (Karim et al., 2018) and the offsets in this case were found to vary from 1.2 to $5.7 \mathrm{~m}$. Ignoring those variations would have produced a distorted surface unsuitable for hydrodynamic modelling. A spatially varying offset surface was created by manually measuring differences at about thirty points around the boundary and interpolating those differences to a difference surface covering the entire area of interest. Once this difference surface was applied to DEM-H, the blending process described in this paper was applied to correct for the fine-scale differences at the boundary.

The extension of the difference surface could be repeated more times to extend the region of the smooth transition. When extended to large enough distances, this smoothing can treat the broader scale differences due to spatially varying systematic errors as well as the fine scale differences due to resolution and local smoothing. Extending the smoothing to large distances increases the computation time, so that approach was not attempted for this project, but that effect can be avoided by generalizing the surface (increasing the cell size) as the smoothing progresses, as was done in the MrVBF and multiscale smoothing methods (Gallant and Dowling, 2003; Gallant, 2011). 


\section{REFERENCES}

Karim, F., Peña-Arancibia, J., Ticehurst, C., Marvanek, S., Gallant, J., Hughes, J., Dutta, D., Vaze, J., Petheram, C., Seo, L. and Kitson, S. (2018). Floodplain inundation mapping and modelling for the Fitzroy, Darwin and Mitchell catchments. CSIRO Technical Report. DOI 10.25919/5b50dfb6c7c0e.

Gallant, J.C. (2011). Adaptive smoothing for noisy DEMs. Geomorphometry 2011. J.P. Wilson, M. Gould, I. S. Evans and T. Hengl. Redlands, California. http://geomorphometry.org/Gallant2011 (accessed 28 July 2019).

Gallant, J.C. and Dowling, T.I. (2003) A multi-resolution index of valley bottom flatness for mapping depositional areas. Water Resources Research 39(12): 1347-1360.

Gallant, J.C., Dowling, T.I., Read, A.M., Wilson, N., Tickle, P., Inskeep, C. (2011) 1 second SRTM Derived Digital Elevation Models User Guide. Geoscience Australia https://d28rz98at9flks.cloudfront.net/72759/1secSRTM_Derived_DEMs_UserGuide_v1.0.4.pdf (accessed 27 July 2019).

Intergovernmental Committee on Surveying and Mapping (2014). Geocentric datum of Australia technical manual, version 2.4. https://www.icsm.gov.au/sites/default/files/2017-09/gda-v_2.4_0.pdf (accessed 27 July 2019).

Leitāo, J. P., Prodanović, D. and Maksimović, Č. (2016). Improving merge methods for grid-based digital elevation models. Computers \& Geosciences 88: 115-131. 